\title{
Coulomb corrections to the extrinsic spin-Hall effect of a two-dimensional electron gas
}

\author{
E. M. Hankiewicz and G. Vignale* \\ Department of Physics and Astronomy, University of Missouri, Columbia, Missouri 65211, USA \\ (Received 9 August 2005; revised manuscript received 3 November 2005; published 30 March 2006)
}

\begin{abstract}
We develop the microscopic theory of the extrinsic spin-Hall conductivity of a two-dimensional electron gas, including skew-scattering, side-jump, and Coulomb interaction effects. We find that while the spin-Hall conductivity connected with the side jump is independent of the strength of electron-electron interactions, the skew-scattering term is reduced by the spin-Coulomb drag, so the total spin current and the total spin-Hall conductivity are reduced for typical experimental mobilities. Further, we predict that in paramagnetic systems the spin-Coulomb drag reduces the spin accumulations in two different ways: (i) directly through the reduction of the skew-scattering contribution, and (ii) indirectly through the reduction of the spin diffusion length. Explicit expressions for the various contributions to the spin-Hall conductivity are obtained using an exactly solvable model of the skew scattering.
\end{abstract}

DOI: 10.1103/PhysRevB.73.115339

PACS number(s): 72.25.Dc, 73.43.-f, 72.25.Rb, 85.75.-d

\section{INTRODUCTION}

There has recently been a strong revival of interest in the phenomenon of the spin-Hall effect in the context of semiconductor spintronics. ${ }^{1}$ Two different forms of this phenomenon have been identified: an extrinsic spin-Hall effect, ${ }^{2-5}$ which is driven by spin-orbit scattering with impurities, and an intrinsic one, ${ }^{6-8}$ which is due to spin-orbit effects in the band structure. In both forms, the phenomenon consists of the appearance of a transverse spin current-say a $z$-spin current in the $y$ direction-when the electron gas is driven by an electric field in the $x$ direction. The physical manifestations of this spin current are still an active subject of study and controversy. ${ }^{9}$ However, it is now believed that the spinHall effect should lead to transverse spin accumulation when the flow of the spin current is suppressed by an appropriate gradient of spin-dependent electrochemical potential. Recently, the spin-Hall effect has been experimentally observed in Kerr rotation experiments in three-dimensional (3D) and two-dimensional (2D) $n$-doped GaAs ${ }^{10,11}$ and in a $p$ - $n$ junction light emitting diode (LED) realized in a twodimensional hole system. ${ }^{12}$ However, there is still a debate on the origin (extrinsic or intrinsic) of the experimentally observed spin accumulation. ${ }^{13-17}$

In this paper we focus exclusively on the theory of the extrinsic effect in a two-dimensional electron gas (2DEG). It has long been realized that the extrinsic spin-Hall current is the sum of two contributions. ${ }^{18}$ The first contribution (commonly known as the "skew-scattering" mechanism" ${ }^{19,20}$ ) arises from the asymmetry of the electron-impurity scattering in the presence of spin-orbit interactions: ${ }^{21}$ electrons that are drifting in the $+x$ direction under the action of an electric field are more likely to be scattered to the left than to the right if, say, their spin is up, while the reverse is true if their spin is down. This generates a net $z$-spin current in the $y$ direction. The second contribution (the "side-jump" mechanism ${ }^{18,22-24}$ ) is caused by the anomalous relationship between the physical and the canonical position operator [see Eq. (6) below]. This again leads to a finite spin current in the $y$ direction. The skew-scattering and side-jump contributions were widely discussed in the context of the anomalous Hall effect $^{18-20,22-26}$ in magnetic materials. The skew-scattering contribution was first studied by Smit ${ }^{19,20}$ while the sidejump contribution was introduced by Berger. ${ }^{22,23}$ The theory for both effects has been also discussed recently in several excellent papers, both for the extrinsic ${ }^{13,16,27}$ and the intrinsic cases. $^{28-31}$

In this paper, in addition to the previously considered skew-scattering and side-jump contributions, we also include the Coulomb interaction effects. The main effect of interactions on the spin transport originates from the friction between spin-up and spin-down electrons moving with different drift velocities, the so called spin-Coulomb drag (SCD) effect. ${ }^{32-34}$ We show that while the spin-Hall conductivity associated with the side-jump term is independent of the strength of electron-electron interactions, the skew-scattering part is reduced by the spin-Coulomb drag, so the absolute value of the spin-Hall conductivity (and hence the spin-Hall current) is reduced for experimentally accessible parameters. Since the SCD has been predicted (and recently observed ${ }^{35}$ ) to be a rather significant contribution to the overall resistivity in high mobility electronic systems, we think it is important to include it in the description of the spin-Hall effect, and we show here how this is done. Moreover, we predict that SCD in paramagnetic materials will reduce the spin accumulations through the reduction of the skew-scattering resistivity as well as the spin-diffusion length. Also, we present in the Appendix a simple model for electron-impurity scattering which can be solved exactly, leading to an analytical determination of scattering rates as well as side-jump and skewscattering contributions to the spin-Hall conductivity.

This paper is organized as follows: In Sec. II the Hamiltonian and the Boltzmann equation are presented; in Sec. III the skew-scattering contribution to the conductivity is derived; in Sec. IV we use a force-balance argument to calculate the side-jump contribution; in Sec. V the contributions of spin-Coulomb drag and spin-flip processes are included; and in Sec. VI the spin accumulation in the presence of skewscattering, side-jump, and electron-electron interactions is calculated. We summarize the paper in Sec. VII. 


\section{HAMILTONIAN AND BOLTZMANN EQUATION}

We consider a strictly two-dimensional electron gas (2DEG) that lies in the $x-y$ plane. The Hamiltonian is

$$
H=H_{0}+H_{s o}+H_{c}+H_{E},
$$

where

$$
H_{0}=\sum_{i}\left[\frac{p_{i}^{2}}{2 m}+V_{e i}\left(\vec{r}_{i}\right)\right]
$$

is the noninteracting Hamiltonian ( $m$ being the effective mass of the conduction band), including the electric potential $V_{e i}(\vec{r})$ generated by randomly distributed impurities,

$$
H_{c}=\frac{1}{2} \sum_{i \neq j} \frac{e^{2}}{\epsilon_{b}\left|\vec{r}_{i}-\vec{r}_{j}\right|},
$$

is the electron-electron interaction (screened by the background dielectric constant $\epsilon_{b}$ ),

$$
H_{s o}=\alpha \sum_{i}\left\{\vec{p}_{i} \times\left[\vec{\nabla}_{i} V_{e i}\left(\vec{r}_{i}\right)+\vec{\nabla}_{i} V_{e e}^{i}\right]\right\} \cdot \vec{\sigma}_{i}
$$

is the spin-orbit interaction (SOI) induced by the electric potential of the impurities, $V_{e i}\left(\vec{r}_{i}\right)$, and of the other electrons $\vec{V}_{e e}^{i}=\sum_{j \neq i} \frac{e^{2}}{\epsilon_{b} \mid \vec{r}_{i}-\vec{r}_{j}}$, and finally

$$
H_{E}=\sum_{i}\left\{e \vec{E} \cdot \vec{r}_{i}+e \alpha\left(\vec{p}_{i} \times \vec{E}\right) \cdot \vec{\sigma}_{i}\right\}
$$

is the interaction with the external electric field $\vec{E}$.

The various spin-orbit terms appearing in the Hamiltonian can all be shown to arise from a single basic fact, namely, the change in form of the physical position operator under a transformation that eliminates the coupling between the conduction band in which the electrons of interest reside, and the spin-orbit-split valence band. If we denote by $\vec{r}_{i}$ the canonical position operator of the $i$ th electron, then the physical position operator is given by

$$
\vec{r}_{\text {phys }, i}=\vec{r}_{i}-\alpha\left(\vec{p}_{i} \times \vec{\sigma}_{i}\right),
$$

and correspondingly the velocity operator is

$$
\vec{v}_{i}=-\frac{i}{\hbar}\left[\vec{r}_{p h y s}, \hat{H}\right]=\frac{\vec{p}_{i}}{m}+2 \alpha\left[\vec{\nabla}_{i} V_{e i}\left(\vec{r}_{i}\right)+\vec{\nabla}_{i} V_{e e}^{i}+e \vec{E}\right] \times \vec{\sigma}_{i} .
$$

The spin-orbit "coupling constant" $\alpha$ takes into account the effective SOI induced by the valence bands (heavy holes, light holes, and split-off band) on conduction electrons in the framework of the eight-band Kane model. Within this model one finds ${ }^{36}$

$$
\alpha=\frac{\hbar P^{2}}{3 m_{e}^{2}}\left[\frac{1}{E_{g}^{2}}-\frac{1}{\left(E_{g}+\Delta_{S O}\right)^{2}}\right],
$$

where $E_{g}$ is the gap energy between conduction and heavy/ light holes bands, $\Delta_{S O}$ is the splitting energy between heavy/ light holes and split-off bands, $P$ is the matrix element of the momentum operator between the conduction and the valence-band edges, and $m_{e}$ is the bare electron mass. Using values of the parameters appropriate for the 2DEG in $\mathrm{Al}_{0.1} \mathrm{Ga}_{0.9} \mathrm{As}$ (Ref. ${ }^{11}$ ) with a band mass $m=0.074 m_{e}$ we find $\alpha \hbar=4.4 \AA^{2}$. In this paper we treat the spin-orbit interaction to the first order in $\alpha$, which is justified by the smallness of the parameter $\alpha \hbar / a_{B}^{2}$, where $a_{B} \simeq 100 \AA$ is the effective Bohr radius.

Notice that the canonical positions $\vec{r}_{i}$ and the canonical momenta $\vec{p}_{i}$ of the particles are vectors in the $x-y$ plane, and so is the $\vec{\nabla}$ operator. Therefore $\vec{p} \times\left[\vec{\nabla}_{i} V_{e i}\left(\vec{r}_{i}\right)+\vec{\nabla}_{i} V_{e e}^{i}\right]$ is a vector in the $z$ direction, and the spin-orbit interaction conserves the $z$ component of the spin of each electron. This nice feature of our strictly 2D model allows a particularly simple analysis of the spin-Hall effect without sacrificing any essential features of the spin-orbit interaction. Processes that flip the $z$ component of the spin will be considered separately (see Sec. V).

We begin to exploit the conservation of $\sigma_{z}$ by defining the quasiclassical one-particle distribution function $f_{\sigma}(\vec{r}, \vec{k}, t)$, i.e., the probability of finding an electron with $z$ component of the spin $S_{z}=(\hbar / 2) \sigma$, with $\sigma= \pm 1$, at position $\vec{r}$ with momentum $\vec{p}=\hbar \vec{k}$ at the time $t$. In this paper we focus on spatially homogeneous steady-state situations, in which $f_{\sigma}$ does not depend on $\vec{r}$ and $t$ (for a discussion of nonhomogeneous spin accumulation effects, see Sec. VI). We write

$$
f_{\sigma}(\vec{r}, \vec{k}, t)=f_{0 \sigma}\left(\epsilon_{k}\right)+f_{1 \sigma}(\vec{k}),
$$

where $f_{0 \sigma}\left(\epsilon_{k}\right)$ is the equilibrium distribution function-a function of the free particle energy $\epsilon_{k}=\left(\hbar^{2} k^{2}\right) / 2 m$-and $f_{1 \sigma}(\vec{k})$ is a small deviation from equilibrium induced by the application of steady electric fields $\vec{E}_{\sigma}(\sigma= \pm 1)$ which couple independently to each of the two spin components. Then to first order in $\vec{E}_{\sigma}$ the Boltzmann equation takes the form

$$
-e \vec{E}_{\sigma} \cdot \frac{\hbar \vec{k}}{m} f_{0 \sigma}^{\prime}\left(\epsilon_{k}\right)=\dot{f}_{1 \sigma}(\vec{k})_{c},
$$

where $\dot{f}_{1 \sigma}(\vec{k})_{c}$ is the first order in $\vec{E}_{\sigma}$ part of the collisional time derivative $\dot{f}_{\sigma}(\vec{k})_{c}$ due to different scattering processes such as electron-impurity scattering, electron-electron scattering, and spin-flip scattering. As usual, $\dot{f}_{\sigma}(\vec{k})_{c}$ is written as the difference of an in-scattering term and an out-scattering term. For example, in the case of spin-conserving electronimpurity scattering, one has

$$
\dot{f}_{\sigma}(\vec{k})_{c, i m p}=-\sum_{\vec{k}^{\prime}}\left[W_{\vec{k}^{\prime} \sigma} f_{\sigma}(\vec{k})-W_{\vec{k}^{\prime} \vec{k} \sigma} f_{\sigma}\left(\vec{k}^{\prime}\right)\right] \delta\left(\widetilde{\epsilon}_{k \sigma}-\widetilde{\epsilon}_{k^{\prime} \sigma}\right),
$$

where $W_{\vec{k} \vec{k}^{\prime} \sigma}$ is the scattering rate for a spin- $\sigma$ electron to go from $\vec{k}$ to $\vec{k}^{\prime}$, and $\widetilde{\epsilon}_{k \sigma}$ is the particle energy, including the additional spin-orbit interaction due to the electric field $\vec{E}_{\sigma}$.

The last point is absolutely vital for a correct accounting of the "side-jump" contribution. We must use

$$
\tilde{\boldsymbol{\epsilon}}_{k \sigma}=\epsilon_{k}+2 e \alpha \hbar \sigma\left(\vec{E}_{\sigma} \times \hat{z}\right) \cdot \vec{k},
$$

where the second term on the right-hand side differs by a factor of 2 from what one would surmise from the intuitive 
expression $\epsilon_{p}+e \mathbf{E} \cdot \mathbf{r}_{\text {phys. }}$. Why? The reason is that the $\delta$ function in Eq. (10) expresses the conservation of energy in a scattering process. This is a time-dependent process; therefore, the correct expression for the change in position of the electron $\Delta \mathbf{r}_{\text {phys }}$ must be calculated as the integral of the velocity over time. From the commutator of the physical position operator with the Hamiltonian we easily find

$$
\vec{v}_{i \sigma}=\frac{\vec{p}_{i}}{m}+2 \alpha \sigma\left[\vec{\nabla}_{i} V_{e i}\left(\vec{r}_{i}\right)+\vec{\nabla}_{i} V_{e e}^{i}+e \vec{E}\right] \times \hat{z},
$$

where the term in the square brackets is (minus) the total force acting on the $i$ th electron. The time integral of this term over the duration of the collision (be it an electron-impurity or an electron-electron collision) gives the change in momentum $\Delta \vec{p}$ during the collision. Thus we see that the change in position is $\Delta \vec{r}_{\text {phys }}=-2 \alpha \sigma \Delta \vec{p} \times \hat{z}$ (this is the so-called side jump) hence, the change in energy is correctly given by Eq. (11).

Kohn and Luttinge ${ }^{37}$ have shown that the above form of the collision integral is correct up to third order in the strength of the electron-impurity approximation; this is one order higher than the Born approximation and should therefore be sufficient to capture the skewdness of the scattering probability, which arises from terms beyond the Born approximation. Notice that the collision integral does not contain the tempting but ultimately incorrect "Pauli-blocking" factors $1-f_{\sigma}\left(\vec{k}^{\prime}\right)$.

Similarly, the electron-electron contribution to the collisional derivative has the form ${ }^{34}$

$$
\begin{aligned}
\dot{f}_{\sigma}(\vec{k})_{c, e-e} \simeq & -\sum_{\vec{k}^{\prime} \vec{p} \vec{p}^{\prime}} W_{C}\left(\vec{k} \sigma, \vec{p}-\sigma ; \vec{k}^{\prime} \sigma, \vec{p}^{\prime}-\sigma\right)\left\{f_{\sigma}(\vec{k}) f_{-\sigma}(\vec{p})\right. \\
& \times\left[1-f_{\sigma}\left(\vec{k}^{\prime}\right)\right]\left[1-f_{-\sigma}\left(\vec{p}^{\prime}\right)\right]-f_{\sigma}\left(\vec{k}^{\prime}\right) f_{-\sigma}\left(\vec{p}^{\prime}\right) \\
& \left.\times\left[1-f_{\sigma}(\vec{k})\right]\left[1-f_{-\sigma}(\vec{p})\right]\right\} \delta_{\vec{k}+\vec{p}, \vec{k}^{\prime}+\vec{p}^{\prime}} \\
& \times \delta\left(\widetilde{\epsilon}_{k \sigma}+\widetilde{\epsilon}_{p-\sigma}-\widetilde{\epsilon}_{k^{\prime} \sigma}-\widetilde{\epsilon}_{p^{\prime}-\sigma}\right),
\end{aligned}
$$

where $W_{C}\left(\vec{k} \sigma, \vec{p}-\sigma ; \vec{k}^{\prime} \sigma, \vec{p}^{\prime}-\sigma\right)$ is the electron-electron scattering rate from $\vec{k} \sigma, \vec{p}-\sigma$ to $\vec{k}^{\prime} \sigma, \vec{p}^{\prime}-\sigma$, and the Pauli factors $f_{\sigma}(\vec{k}), 1-f_{\sigma}\left(\vec{k}^{\prime}\right)$ etc. ensure that the initial states are occupied and the final states are empty as required by Pauli's exclusion principle. Notice that, for our purposes, only collisions between electrons of opposite spins are relevant, since collision between same-spin electrons conserve the total momentum of each spin component. Accordingly, only the former have been retained in Eq. (13).

\section{SKEW SCATTERING}

Let us, at first, neglect the electron-electron interaction. From the general theory developed, for instance, in Ref. 38, one can easily deduce that the scattering amplitude from one impurity in two dimensions has the form

$$
f_{\vec{k} \vec{k}^{\prime}, \sigma}=A_{\vec{k} \vec{k}^{\prime}}+\sigma B_{\vec{k} \vec{k}^{\prime}}\left(\hat{k} \times \hat{k}^{\prime}\right)_{z},
$$

where $A_{\vec{k} \vec{k}^{\prime}}$ and $B_{\vec{k} \vec{k}^{\prime}}$ are complex scattering amplitudes, and $\hat{k}$ and $\hat{k}^{\prime}$ are the unit vectors in the directions of $\vec{k}$ and $\vec{k}^{\prime}$ respectively. The second term on the right-hand side of Eq. (14) is due to the spin-orbit interaction. Squaring the scattering amplitude and multiplying by the number $N_{i}$ of independent scattering centers we arrive at the following expression for the scattering rate from $\vec{k}$ to $\vec{k}^{\prime}$ :

$$
W_{\vec{k} \vec{k}^{\prime}, \sigma}=\left[W_{\vec{k} k^{\prime}}^{s}+\sigma W_{\vec{k} \vec{k}^{\prime}}^{a}\left(\hat{k} \times \hat{k}^{\prime}\right)_{z}\right] \delta\left(\epsilon_{k}-\epsilon_{k^{\prime}}\right),
$$

where

$$
W_{\vec{k} \vec{k}^{\prime}}^{S}=N_{i}\left[\left|A_{\vec{k} \vec{k}^{\prime}}\right|^{2}+\left|B_{\vec{k} \vec{k}^{\prime}}\right|^{2}\right],
$$

and

$$
W_{\vec{k} \vec{k}^{\prime}}^{a}=2 N_{i} R e\left[A_{\vec{k} \vec{k}^{\prime}} B_{\vec{k} \vec{k}^{\prime}}^{*}\right] .
$$

Here and in the following $\epsilon_{k} \equiv\left(\hbar^{2} k^{2}\right) / 2 m$ is the free particle energy. The second term in the square brackets of Eq. (15) depends on the spin $(\sigma=+1$ or -1 for up or down spins, respectively) and on the chirality of the scattering [i.e., the sign of $\left.\left(\hat{k} \times \hat{k}^{\prime}\right)_{z}\right]$. We will refer to this as the skew-scattering term. It should be noted that for a centrally symmetric scattering potential-the only case we are going to consider in this paper- $W_{\vec{k} \vec{k}^{\prime}}^{s}$ and $W_{\vec{k} \vec{k}^{\prime}}^{a}$ depend only on the magnitude of the vectors $\vec{k}$ and $\vec{k}^{\prime}$, which are equal by energy conservation, and on the angle $\theta$ between them. Furthermore, they are both symmetric under interchange of $\vec{k}$ and $\vec{k}^{\prime}$-the antisymmetry of the skew scattering being explicitly brought in by the factor $\left(\hat{k} \times \hat{k}^{\prime}\right)_{z}=\sin \theta$. Thus, in the following, we will often write $W_{\vec{k} k^{\prime}}^{s / a} \equiv W^{s / a}(k, \theta)$, where $W^{s / a}(k, \theta)$ are even functions of $\theta$. Notice that the skew scattering term vanishes when the scattering is treated in the second-order Born approximation. ${ }^{38}$ Indeed, within this approximation $A_{\vec{k} \vec{k}^{\prime}}$ is purely real and $B_{\vec{k} \vec{k}^{\prime}}$ is purely imaginary, so $W_{\vec{k} \vec{k}^{\prime}}^{a}$ is zero.

The linearized Boltzmann equation can be solved exactly under the assumption that $W_{\vec{k} \vec{k}^{\prime}}^{s}$ and $W_{\vec{k} \vec{k}^{\prime}}^{a}$ depend only on the energy $\epsilon_{k}=\epsilon_{k^{\prime}}$ and on the angle $\theta$ between $\vec{k}$ and $\vec{k}^{\prime}$. The solution has the form

$$
f_{\sigma}(\vec{k})=f_{0 \sigma}\left(\epsilon_{k}\right)-f_{0 \sigma}^{\prime}\left(\epsilon_{k}\right) \hbar \vec{k} \cdot \vec{V}_{\sigma}(k),
$$

where $\vec{V}_{\sigma}(k)$ is proportional to the electric field. In view of Eq. (11) it is convenient to expand

$$
f_{0 \sigma}\left(\epsilon_{k}\right)=f_{0 \sigma}\left(\widetilde{\epsilon}_{k \sigma}\right)-f_{0 \sigma}^{\prime}\left(\epsilon_{k}\right)\left(\widetilde{\epsilon}_{k \sigma}-\epsilon_{k}\right) .
$$

so that our ansatz (18) takes the form

$$
\begin{aligned}
f_{\sigma}(\vec{k})= & f_{0 \sigma}\left(\widetilde{\epsilon}_{k \sigma}\right)-2 f_{0 \sigma}^{\prime}\left(\epsilon_{k}\right) e \alpha \hbar \sigma\left(\vec{E}_{\sigma} \times \hat{z}\right) \cdot \vec{k} \\
& -f_{0 \sigma}^{\prime}\left(\epsilon_{k}\right) \hbar \vec{k} \cdot \vec{V}_{\sigma}(k) .
\end{aligned}
$$

The advantage of this form is that the "zero-order term" $f_{0 \sigma}\left(\widetilde{\epsilon}_{k \sigma}\right)$ makes no contribution to the collision integral (10). Then, making use of Eq. (15) and discarding terms proportional to $\alpha W^{a}$ (which are small since $W^{a}$ itself is proportional to $\alpha$ ) we arrive at the following form for the linearized collision integral: 


$$
\begin{aligned}
\dot{f}_{1 \sigma}(\vec{k})_{c, i m p}= & -\sum_{\vec{k}^{\prime}} W_{\vec{k} \vec{k}^{\prime}}^{s}\left\{f_{1 \sigma}(\vec{k})-f_{1 \sigma}\left(\vec{k}^{\prime}\right)\right\} \delta\left(\epsilon_{k}-\epsilon_{k^{\prime}}\right) \\
& -\sigma \sum_{\vec{k}^{\prime}} W_{\vec{k} \vec{k}^{\prime}}^{a}\left(\hat{k} \times \hat{k}^{\prime}\right)_{z}\left\{f_{1 \sigma}(\vec{k})+f_{1 \sigma}\left(\vec{k}^{\prime}\right)\right\} \\
& \times \delta\left(\epsilon_{k}-\epsilon_{k^{\prime}}\right)+2 \sigma \sum_{\vec{k}^{\prime}} W_{\vec{k} \vec{k}^{\prime}}^{s} f_{0 \sigma}^{\prime}\left(\epsilon_{k}\right) e \alpha \hbar \\
& \times\left(\vec{E}_{\sigma} \times \hat{z}\right) \cdot\left(\vec{k}-\vec{k}^{\prime}\right) \delta\left(\epsilon_{k}-\epsilon_{k^{\prime}}\right),
\end{aligned}
$$

where $f_{1 \sigma}(\vec{k}) \equiv-f_{0 \sigma}^{\prime}\left(\epsilon_{k}\right) \hbar \vec{k} \cdot \vec{V}_{\sigma}(k)$ is the deviation of the distribution function from unpertubed equilibrium.

At low temperature, it is not necessary to take into account the full $k$ dependence of $\vec{V}_{\sigma}(k)$, since the derivative of the Fermi distribution $f_{0 \sigma}^{\prime}\left(\epsilon_{k}\right)$ restricts the range of $k$ to the vicinity of the Fermi wave vectors $k_{F \sigma}$. Thus we replace the function $V_{\sigma}(k)$ by a constant $V_{\sigma}$, and determine $V_{\sigma}$ from the consistency condition

$$
-e \sum_{\vec{k}} \frac{\hbar \vec{k}}{m}\left[\vec{E}_{\sigma} \cdot \frac{\hbar \vec{k}}{m}\right] f_{0 \sigma}^{\prime}\left(\epsilon_{k}\right)=\sum_{\vec{k}} \frac{\hbar \vec{k}}{m} \dot{f}_{1 \sigma}(\vec{k})_{c, i m p} .
$$

Substituting the collision integral from Eq. (21) on the righthand side of this equation and moving its last term to the left-hand side we arrive at

$$
\begin{aligned}
& \frac{e n_{\sigma}}{m} \vec{E}_{\sigma}-2 e \sigma \sum_{\vec{k} k^{\prime}} \frac{\hbar^{2} \vec{k}}{m}\left[( \vec { k } - \vec { k } ^ { \prime } ) W _ { \vec { k } \vec { k } ^ { \prime } } ^ { s } \alpha ( \vec { E } _ { \sigma } \times \hat { z } ) f _ { 0 \sigma } ^ { \prime } ( \epsilon _ { k } ) \delta \left(\epsilon_{k}\right.\right. \\
& \left.\left.-\epsilon_{k^{\prime}}\right)\right]=\sum_{\vec{k} \vec{k}^{\prime}} W_{\vec{k} \vec{k}^{\prime}}^{s} \frac{\hbar^{2} \vec{k}}{m}\left(\vec{k}-\vec{k}^{\prime}\right) \cdot \vec{V}_{\sigma} f_{0 \sigma}^{\prime}\left(\epsilon_{k}\right) \delta\left(\epsilon_{k}-\epsilon_{k^{\prime}}\right) \\
& +\sigma \sum_{\vec{k} \vec{k}^{\prime}} W_{\vec{k} \vec{k}^{\prime}}^{a} \frac{\hbar^{2} \vec{k}}{m}\left(\hat{k} \times \hat{k}^{\prime}\right)_{z}\left(\vec{k}+\vec{k}^{\prime}\right) \cdot \vec{V}_{\sigma} f_{0 \sigma}^{\prime}\left(\epsilon_{k}\right) \delta\left(\epsilon_{k}-\epsilon_{k^{\prime}}\right),
\end{aligned}
$$

where $n_{\sigma}=k_{F \sigma}^{2} / 4 \pi$ is the density of $\sigma$-spin carriers and $k_{F \sigma}$ is the corresponding Fermi wave vector. The first term on the right-hand side of Eq. (23) is parallel to $\vec{V}_{\sigma}$, while the second term is orthogonal to it. Then a simple calculation leads to the following expression for $\vec{V}_{\sigma}$ in terms of the electric field:

$$
-\frac{e}{m} \vec{E}_{\sigma}-\frac{2 e \alpha \sigma\left(\vec{E}_{\sigma} \times \hat{z}\right)}{\tau_{\sigma}}=\frac{\vec{V}_{\sigma}}{\tau_{\sigma}}+\sigma \frac{\vec{V}_{\sigma} \times \hat{z}}{\tau_{\sigma}^{s s}},
$$

and its inverse to first order in $\alpha$ is

$$
\vec{V}_{\sigma}=\frac{-e \tau_{\sigma}}{m}\left[\vec{E}_{\sigma}-\sigma \frac{\tau_{\sigma}}{\tau_{\sigma}^{s s}} \vec{E}_{\sigma} \times \hat{z}\right]-2 e \alpha \sigma\left(E_{\sigma} \times \hat{z}\right),
$$

where

$$
\frac{1}{\tau_{\sigma}}=-\frac{m \mathcal{A}}{4 \pi^{2} \hbar^{2} \epsilon_{F \sigma}} \int_{0}^{\infty} d \epsilon \epsilon f_{0 \sigma}^{\prime}(\epsilon) \int_{0}^{2 \pi} d \theta W^{s}(\epsilon, \theta)(1-\cos \theta),
$$

and

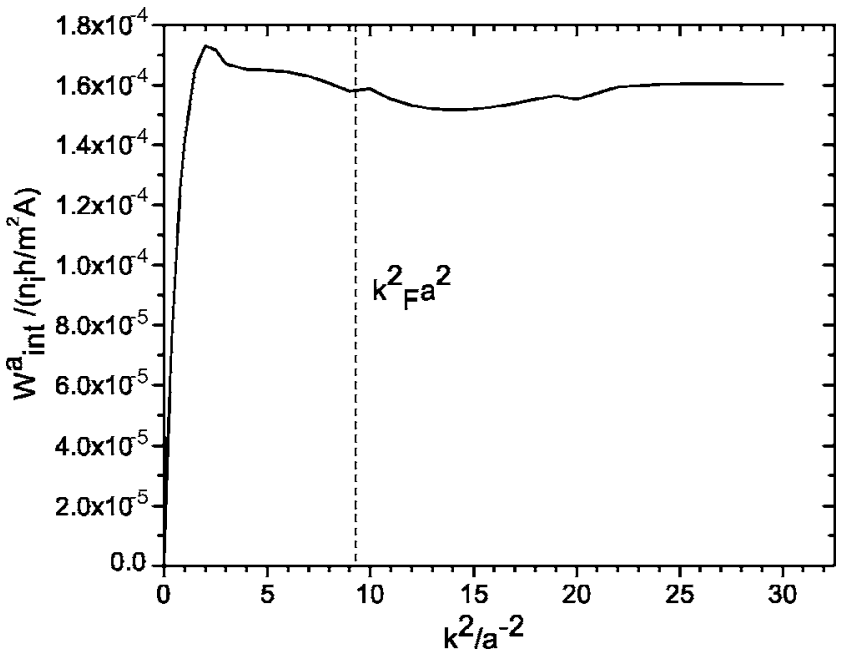

FIG. 1. Antisymmetric scattering rate $W_{i n t}^{a}$ $=\int_{0}^{2 \pi} d \theta W^{a}(\epsilon, \theta) \sin ^{2} \theta$ in units of $n_{i} h / m^{2} \mathcal{A}$ [see Eq. (A22)] as a function of $k^{2}$ for a model circular well attractive potential $V_{0}=$ $-5 \mathrm{meV}$ and radius $a=9.45 \mathrm{~nm}$ (described in the Appendix). We choose the parameters typical for the experimental 2DEG confined in $\mathrm{Al}_{0.1} \mathrm{Ga}_{0.9}$ As quantum well, i.e., density of electrons and impurities $n_{2 D}=n_{i}=2.0 \times 10^{12} \mathrm{~cm}^{-2}, \quad m=0.074 \mathrm{~m}_{e}$, and mobility $\bar{\mu}$ $=0.1 \mathrm{~m}^{2} /$ Vs. The effective spin-orbit coupling $\alpha \hbar=4.4 \AA^{2}$ in accordance with (Ref. 36).

$$
\frac{1}{\tau_{\sigma}^{s s}}=-\frac{m \mathcal{A}}{4 \pi^{2} \hbar^{2} \epsilon_{F \sigma}} \int_{0}^{\infty} d \epsilon \epsilon f_{0 \sigma}^{\prime}(\epsilon) \int_{0}^{2 \pi} d \theta W^{a}(\epsilon, \theta) \sin ^{2} \theta .
$$

In the above equations $\epsilon_{F \sigma}=k_{F \sigma}^{2} \hbar^{2} / 2 m$ is the Fermi energy for spin $\sigma$. In the limit of zero temperature the derivative of the Fermi function reduces to $f_{0 \sigma}^{\prime}(\epsilon) \simeq-\delta\left(\epsilon-\epsilon_{F \sigma}\right)$ and the above formulas simplify as follows:

$$
\frac{1}{\tau_{\sigma}} \stackrel{T \rightarrow 0}{\simeq} \frac{m \mathcal{A}}{4 \pi^{2} \hbar^{2}} \int_{0}^{2 \pi} d \theta W^{S}\left(k_{F}, \theta\right)(1-\cos \theta),
$$

and

$$
\frac{1}{\tau_{\sigma}^{s s}} \stackrel{T \rightarrow 0}{\simeq} \frac{m \mathcal{A}}{4 \pi^{2} \hbar^{2}} \int_{0}^{2 \pi} d \theta W^{a}\left(k_{F}, \theta\right) \sin ^{2} \theta .
$$

Figure 1 shows the antisymmetric scattering rate $\int_{0}^{2 \pi} d \theta W^{a}(\epsilon, \theta) \sin ^{2} \theta$ calculated numerically using Eq. (A22) for a model impurity potential Eq. (A1) presented in Appendix, and for the typical experimental parameters. ${ }^{11}$

\section{SPIN-HALL CURRENT AND SIDE JUMP}

The quantity $\vec{V}_{\sigma}$ obtained in Eq. (25), determines the nonequilibrium distribution, according to Eq. (18). We now use this distribution to calculate the current density. In order to do this correctly, however, we must remember that the spinorbit interaction alters the relation between the velocity and the canonical momentum. The correct expression for the velocity is given in Eq. (7) and in the absence of electronelectron interactions takes the form 


$$
\vec{v}_{i}=\frac{\vec{p}_{i}}{m}+2 \alpha\left[\vec{\nabla} V_{e i}\left(\vec{r}_{i}\right)+e \vec{E}\right] \times \vec{\sigma}_{i}
$$

The second term on the right-hand side of this equation contains the net force resulting from the combined action of the external electric field and the impurity potential on the $i$ th electron. This force must vanish when averaged in a nonequilibrium steady-state ensemble, since the average value of the momentum must be stationary. Also, the same is true in the presence of electron-electron interactions, provided the Coulomb force is included. Thus, we arrive at the simple result

$$
\vec{j}_{\sigma}=-e n_{\sigma} \vec{V}_{\sigma}
$$

Combining this with Eq. (25) we obtain the complete relation between electric field and current density

$$
\vec{j}_{\sigma}=\frac{n_{\sigma} e^{2} \tau_{\sigma}}{m}\left[\vec{E}_{\sigma}-\sigma \frac{\tau_{\sigma}}{\tau_{\sigma}^{s}} \vec{E}_{\sigma} \times \hat{z}\right]+2 e^{2} \alpha \sigma n_{\sigma} \vec{E}_{\sigma} \times \hat{z}
$$

The last term on the right-hand side of this expression is known in the literature as the side-jump contribution to the current density. ${ }^{18}$ It comes from the use of $\tilde{\epsilon}_{k}$ rather than $\epsilon_{k}$ in the $\delta$ function of conservation of energy; see discussion in the paragraph following Eq. (11). Inverting Eq. (32) we obtain the following formula for the electric field in terms of the current densities:

$$
\vec{E}_{\sigma}=\rho_{\sigma}^{D} \vec{j}_{\sigma}+\sigma\left[\rho_{\sigma}^{s s}-\lambda_{\sigma} \rho_{\sigma}^{D}\right] \vec{j}_{\sigma} \times \hat{z}
$$

where $\rho_{\sigma}^{D}=m / n_{\sigma} e^{2} \tau_{\sigma}$ is the Drude resistivity, $\rho_{\sigma}^{s s}=m / n_{\sigma} e^{2} \tau_{\sigma}^{s s}$, $\lambda_{\sigma}=2 m \alpha / \tau_{\sigma}$. Hence the resistivity tensor, written in the basis $x_{\uparrow}, y_{\uparrow}, x_{\downarrow}, y_{\downarrow}$, has the following form:

$$
\rho=\left(\begin{array}{cccc}
\rho_{\uparrow}^{D} & \rho_{\uparrow}^{s s}-\lambda_{\uparrow} \rho_{\uparrow}^{D} & 0 & 0 \\
-\rho_{\uparrow}^{s s}+\lambda_{\uparrow} \rho_{\uparrow}^{D} & \rho_{\uparrow}^{D} & 0 & 0 \\
0 & 0 & \rho_{\downarrow}^{D} & -\rho_{\downarrow}^{s s}+\lambda_{\downarrow} \rho_{\downarrow}^{D} \\
0 & 0 & \rho_{\downarrow}^{s s}-\lambda_{\downarrow} \rho_{\downarrow}^{D} & \rho_{\downarrow}^{D}
\end{array}\right) .
$$

The diagonal part of the resistivity reduces to the Drude formula $\rho_{\sigma}^{D}=m /\left(n_{\sigma} e^{2} \tau_{\sigma}\right)$ as expected. The spin-orbit interaction is entirely responsible for the appearance of an off-diagonal (transverse) resistivity. The latter consists of two competing terms associated with side jump $\left(\lambda_{\sigma} \rho_{\sigma}^{D}\right)$ and skew scattering $\left(\rho_{\sigma}^{s S}\right)$, as seen in Eq. (34). Hence our expression for the transverse resistivity is different from the expression presented in previous papers (see for example Ref. 5) where only the side-jump contribution appears in the final formulas. Notice that, at this level of approximation, the resistivity is diagonal in the spin indices.

\section{SPIN-COULOMB DRAG}

Up to this point we have ignored electron-electron scattering processes, as well as scattering processes that might flip the spin of the electrons. As discussed in Ref. 34 these processes are important because they couple the up- and down-spin components of the current density, giving rise to off-diagonal elements, $\rho_{\uparrow \downarrow}^{\alpha \beta}$, of the resistivity tensor. The Coulomb interaction, in particular, leads to the phenomenon of the spin-Coulomb drag (SCD) - a form of friction caused by the relative drift motion of spin-up and spin-down electrons, and the consequent transfer of momentum between them. ${ }^{33-35,39}$ Both Coulomb and spin-flip scattering can be included in our formulation as additional contributions to the collisional derivative $\dot{f}_{1 \sigma}(\vec{k})_{c}$ (see Ref. 34). In particular, the Coulomb contribution is given by Eq. (13). Substituting Eq. (20) into Eq. (13) and including the first-order corrections from electron-electron interactions to the spin-orbit Hamiltonian, we arrive at the following expression for the Coulomb collision integral:

$$
\begin{aligned}
\dot{f}_{\sigma}(\vec{k})_{c, e-e} \simeq & -\frac{1}{k_{B} T} \sum_{\vec{k}^{\prime} \vec{p}^{\prime}} W_{C}\left(\vec{k} \sigma, \vec{p}-\sigma ; \vec{k}^{\prime} \sigma, \vec{p}^{\prime}-\sigma\right)\left[\hbar \vec{V}_{\sigma}-\hbar \vec{V}_{-\sigma}+2 e \alpha \hbar \sigma\left(\vec{E}_{\sigma}+\vec{E}_{-\sigma}\right) \times \hat{z}\right] \cdot\left(\vec{k}-\vec{k}^{\prime}\right) \\
& \times f_{0 \sigma}\left(\epsilon_{k}\right) f_{0-\sigma}\left(\epsilon_{p}\right) f_{0 \sigma}\left(-\epsilon_{k^{\prime}}\right) f_{0-\sigma}\left(-\epsilon_{p^{\prime}}\right) \delta_{\vec{k}+\vec{p}, \vec{k}^{\prime}+\vec{p}^{\prime}} \delta\left(\epsilon_{k \sigma}+\epsilon_{p-\sigma}-\epsilon_{k^{\prime} \sigma}-\epsilon_{p^{\prime}-\sigma}\right),
\end{aligned}
$$

where $T$ is the temperature, $k_{B}$ is the Boltzmann constant, and we have made use of the identity $f_{0 \sigma}\left(\epsilon_{k}\right) f_{0-\sigma}\left(\epsilon_{p}\right)[1$ $\left.-f_{0 \sigma}\left(\epsilon_{k^{\prime}}\right)\right]\left[1-f_{0-\sigma}\left(\epsilon_{p^{\prime}}\right)\right]=\left[1-f_{0 \sigma}\left(\epsilon_{k}\right)\right]\left[1-f_{0-\sigma}\left(\epsilon_{p}\right)\right]$ $\times f_{0 \sigma}\left(\epsilon_{k^{\prime}}\right) f_{0-\sigma}\left(\epsilon_{p^{\prime}}\right)$ for $\epsilon_{k \sigma}+\epsilon_{p-\sigma}-\epsilon_{k^{\prime} \sigma}-\epsilon_{p^{\prime}-\sigma}=0$.
Equation (35) is now inserted into the "consistency condition" (22), and the resulting sum over momenta is expressed in terms of the spin-drag coefficient $\gamma$, i.e., the rate of momentum transfer between up- and down-spin electrons, according to the formula ${ }^{34}$ 


$$
\begin{aligned}
\gamma= & \frac{n}{n_{\sigma} n_{-\sigma}} \sum_{\vec{k} \vec{k}^{\prime} \vec{p} \vec{p}^{\prime}} W_{C}\left(\vec{k} \sigma, \vec{p}-\sigma ; \vec{k}^{\prime} \sigma, \vec{p}^{\prime}-\sigma\right) \\
& \times \frac{\left(\vec{k}-\vec{k}^{\prime}\right)^{2}}{4 m k_{B} T} f_{0 \sigma}\left(\epsilon_{k}\right) f_{0-\sigma}\left(\epsilon_{p}\right) f_{0 \sigma}\left(-\epsilon_{k^{\prime}}\right) f_{0-\sigma}\left(-\epsilon_{p^{\prime}}\right) \\
& \times \delta_{\vec{k}+\vec{p}, \vec{k}^{\prime}+\vec{p}^{\prime}} \delta\left(\epsilon_{k \sigma}+\epsilon_{p-\sigma}-\epsilon_{k^{\prime} \sigma}-\epsilon_{p^{\prime}-\sigma}\right) .
\end{aligned}
$$

Then Eq. (24) for $\vec{V}_{\sigma}$ is modified as follows:

$$
\begin{aligned}
-\frac{e}{m} \vec{E}_{\sigma}-2 \frac{e \alpha \sigma\left(\vec{E}_{\sigma} \times \hat{z}\right)}{\tau_{\sigma}}= & \frac{\vec{V}_{\sigma}}{\tau_{\sigma}}+\sigma \frac{\vec{V}_{\sigma} \times \hat{z}}{\tau_{\sigma}^{s s}}-\frac{n_{-\sigma} \gamma}{n_{\sigma} n e} \vec{j}_{\sigma}+\frac{\gamma}{n e} \vec{j}_{-\sigma} \\
& +2 \gamma \frac{n_{-\sigma}}{n} e \alpha \sigma\left(\vec{E}_{\sigma}+\vec{E}_{-\sigma}\right) \times \hat{z} \\
& +\frac{1}{\tau_{\sigma}^{\prime} n_{\sigma} e} \vec{j}_{-\sigma} .
\end{aligned}
$$

The third and fourth terms on the right-hand side (r.h.s.) of this equation are connected with the SCD and $\gamma$ is the spindrag coefficient calculated in Ref. 39. The fifth term on the r.h.s. of Eq. (37) comes from the side-jump effect in Coulomb scattering. The last term in Eq. (37) is associated with spin-flip collision processes, characterized by the spinrelaxation time $\tau_{\sigma}^{\prime}$. We include it for completeness, even though spin-flip effects are expected to be small in $n$-doped semiconductors. The current is now given by the full velocity operator of Eq. (7), but thanks to the force balance condition in the steady state it reduces again to the simple form

$$
\vec{j}_{\sigma}=-e n_{\sigma} \vec{V}_{\sigma}
$$

Combining Eq. (37) with Eq. (38) leads to the resistivity tensor, which in the basis of $x_{\uparrow}, y_{\uparrow}, x_{\downarrow}, y_{\downarrow}$ has the form

$$
\rho=\left(\begin{array}{cccc}
\rho_{\uparrow}^{D}+\rho^{S D} n_{\downarrow} / n_{\uparrow} & \rho_{\uparrow}^{s S}-\lambda_{\uparrow} \rho_{\uparrow}^{D}+A_{\uparrow}^{\gamma \alpha} & -\rho^{S D}-\rho_{\uparrow}^{\prime} & B_{\uparrow}^{\gamma \alpha} \\
-\rho_{\uparrow}^{s S}+\lambda_{\uparrow} \rho_{\uparrow}^{D}-A_{\uparrow}^{\gamma \alpha} & \rho_{\uparrow}^{D}+\rho^{S D} n_{\downarrow} / n_{\uparrow} & -B_{\uparrow}^{\gamma \alpha} & -\rho^{S D}-\rho_{\uparrow}^{\prime} \\
-\rho^{S D}-\rho_{\downarrow}^{\prime} & -B_{\downarrow}^{\gamma \alpha} & \rho_{\downarrow}^{D}+\rho^{S D} n_{\uparrow} / n_{\downarrow} & -\rho_{\downarrow}^{s S}+\lambda_{\downarrow} \rho_{\downarrow}^{D}-A_{\downarrow}^{\gamma \alpha} \\
B_{\downarrow}^{\gamma \alpha} & -\rho^{S D}-\rho_{\downarrow}^{\prime} & \rho_{\downarrow}^{s S}-\lambda_{\downarrow} \rho_{\downarrow}^{D}+A_{\downarrow}^{\gamma \alpha} & \rho_{\downarrow}^{D}+\rho^{S D} n_{\uparrow} / n_{\downarrow}
\end{array}\right),
$$

where $\rho^{S D}=m \gamma / n e^{2}$ is the spin-Coulomb drag resistivity and $\rho_{\sigma}^{\prime}=m / n_{\sigma} e^{2} \tau_{\sigma}^{\prime}$ (recall that $\lambda_{\sigma}=2 m \alpha / \tau_{\sigma}$ is a dimensionless quantity). $A_{\sigma}^{\gamma \alpha}$ and $B_{\sigma}^{\gamma \alpha}$ represent the terms of the first order in electron-electron coupling $\gamma$ and in SO coupling $\alpha$ and are defined as follows: $A_{\sigma}^{\gamma \alpha}=-\lambda_{\sigma} \rho_{S D} n_{-\sigma} / n_{\sigma}+2 m^{*} \alpha \gamma\left[-n_{-\sigma} \rho_{\sigma}^{D} / n\right.$ $\left.+\left(n_{-\sigma} / n-n_{-\sigma}^{2} / n n_{\sigma}\right) \rho^{S D}\right] \quad$ and $\quad B_{\sigma}^{\gamma \alpha}=\lambda_{\sigma} \rho_{S D}+2 m^{*} \alpha \gamma[$ $\left.-n_{-\sigma} \rho_{-\sigma}^{D} / n+\left(n_{-\sigma} / n-n_{\sigma} / n\right) \rho^{S D}\right]$. Notice that the resistivity satisfies the following symmetry relations:

$$
\begin{gathered}
\rho_{\sigma \sigma}^{\beta \beta^{\prime}}=-\rho_{\sigma \sigma}^{\beta^{\prime} \beta}, \\
\rho_{\sigma-\sigma}^{\beta \beta^{\prime}}=\rho_{-\sigma \sigma}^{\beta^{\prime} \beta},
\end{gathered}
$$

where upper indices $\beta$ and $\beta^{\prime}$ denote directions, and the lower ones spin orientations. From Eq. (39) one clearly sees that the spin-Coulomb drag and spin-flip processes couple the spin components of the longitudinal resistivity. Further, the spin-Coulomb drag corrections to the spin-orbit Hamiltonian ( $\alpha \gamma$ corrections) result in the $\rho_{\sigma-\sigma}^{\beta \beta^{\prime}}$ terms, i.e., in the transverse resistivity which couples opposite spins. Also, the SCD renormalizes the longitudinal spin-diagonal components $\rho_{\sigma-\sigma}^{\beta \beta}$ of the resistivity, in such a way as to satisfy Galilean invariance.

The spin-flip collision processes come as a phenomenological term which could have origin for example in some random magnetic field, which does not appear in the original spin-conserving Hamiltonian [Eq. (1)]. The spin-flip relaxation time, $\tau_{\sigma}^{\prime}$ is given by the microscopic expression ${ }^{34}$

$$
\frac{1}{\tau_{\sigma}^{\prime}}=-\frac{m \mathcal{A}}{8 \pi^{2} \hbar^{2} \epsilon_{F-\sigma}} \int_{0}^{2 \pi} d \theta W_{\sigma,-\sigma}^{s f}\left(\epsilon_{k}, \theta\right) \cos \theta,
$$

where $W_{\sigma,-\sigma}^{s f}$ denotes the spin-flip scattering rate from spin $\sigma$ to the opposite spin orientation $-\sigma .^{45}$ Since the relaxation time for spin-flip processes $\tau^{\prime}$ is very long, ${ }^{34}$ the SCD normally controls the coupling between the spin components, except at the very lowest temperatures (the spin drag rate $\gamma$ vanishes as $T^{2} \ln T$ while the spin-flip rate remains constant). Spin-flip processes will therefore be omitted henceforth.

In a paramagnetic material there is symmetry between upand down-spin densities, mobilities, etc. and one can easily separate the spin and charge degrees of freedom. Then combining the Eq. (37) and Eq. (38) simplifies to

$$
\begin{gathered}
\vec{E}_{c}=\rho^{D} \vec{j}_{c}+2\left(\rho^{s s}-\lambda \rho^{D}-\lambda \rho_{S D}\right) \vec{j}_{s} \times \hat{z}, \\
\vec{E}_{s}=4\left(\rho^{S D}+\rho^{D}\right) \vec{j}_{s}+2\left(\rho^{s s}-\lambda \rho^{D}-\lambda \rho_{S D}\right) \vec{j}_{c} \times \hat{z},
\end{gathered}
$$

where we omit spin-flip processes, and the charge/spin components of the electric field are defined as $\vec{E}_{c}=\left(\vec{E}_{\uparrow}+\vec{E}_{\downarrow}\right) / 2$, $\vec{E}_{s}=\vec{E}_{\uparrow}-\vec{E}_{\downarrow}$, and the charge and spin currents are $\vec{j}_{c}=\vec{j}_{\uparrow}+\vec{j}_{\downarrow}$ and $\vec{j}_{s}=\left(\overrightarrow{j_{\uparrow}}-\overrightarrow{j_{\downarrow}}\right) / 2$, respectively. The spin-Coulomb drag renormalizes the longitudinal resistivity only in the spin 


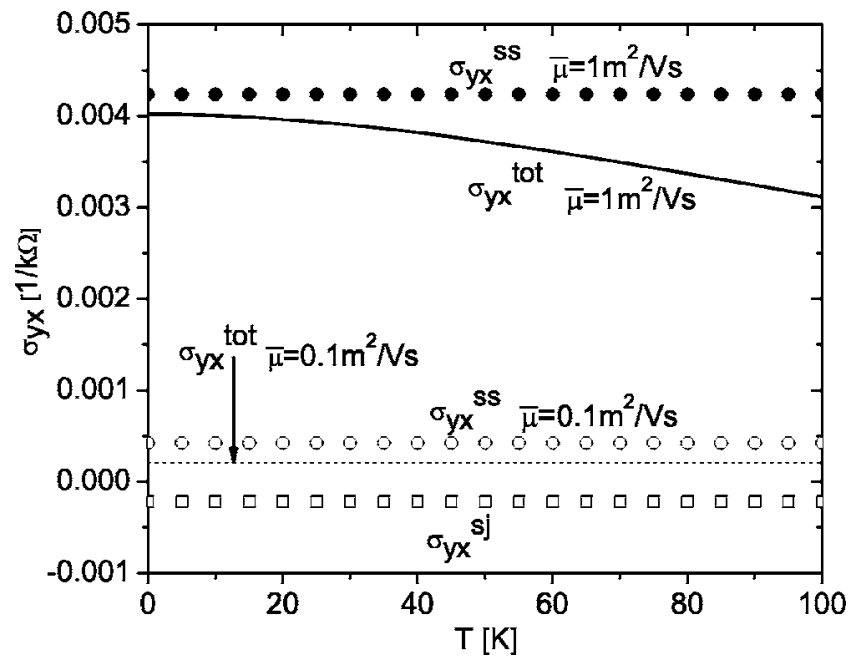

FIG. 2. Spin Hall conductivity as a function of temperature. $\sigma_{y x}^{s j}$ (open squares), $\sigma_{y x}^{s s}$ (open and closed circles) are the side-jump and skew-scattering contributions, respectively, to the conductivity in the absence of electron-electron interactions, and $\sigma_{y x}^{t o t}$ is the total spin conductivity when electron-electron interactions are taken into account. We choose the parameters typical for the experimental 2DEG confined in $\mathrm{Al}_{0.1} \mathrm{Ga}_{0.9}$ As quantum well, i.e., density of electrons and impurities $n_{2 D}=n_{i}=2.0 \times 10^{12} \mathrm{~cm}^{-2}, m=0.074 \mathrm{~m}_{e}$, and two sets of mobilities and relaxations times: $\bar{\mu}=0.1 \mathrm{~m}^{2} / \mathrm{Vs}, \tau=4$ $\times 10^{-5} \mathrm{~ns}, \quad \tau_{s s}=0.02 \mathrm{~ns}, \quad$ and $\bar{\mu}=1 \mathrm{~m}^{2} / \mathrm{Vs} \quad \tau=4 \times 10^{-4} \mathrm{~ns}, \quad \tau_{s s}$ $=0.2$ ns. The effective spin-orbit coupling $\alpha \hbar=4.4 \AA^{2}$ in accordance with Ref. 36. We used the model potential (see Appendix) where an effective impurity radius $a=9.45 \mathrm{~nm}$, the height of attractive impurity potential $V_{0}=-5 \mathrm{meV}$ for $\bar{\mu}=0.1 \mathrm{~m}^{2} / \mathrm{Vs}$, and $V_{0}=$ $-1.6 \mathrm{meV}$ for $\bar{\mu}=1 \mathrm{~m}^{2} / \mathrm{Vs}$.

channel. This is a consequence of the fact that the net force exerted by spin-up electrons on spin-down electrons is proportional to the difference of their drift velocities, i.e., to the spin current. Additionally, the electron-electron corrections to the spin-orbit interactions renormalize the transverse resistivity in the charge and spin channels, so the Onsager relations between spin and charge channels hold. Under the assumption that the electric field is in the $x$ direction has the same value for spin up and spin down we see that Eqs. (43) and (44) yield the following formula for the spin current in $y$ direction:

$$
j_{y}^{s z}=\left[\frac{\rho^{s s} / 2\left(\rho^{D}\right)^{2}}{1+\rho^{S D} / \rho^{D}}-\frac{\lambda}{2 \rho_{D}}\right] E_{x} .
$$

The first term in the square brackets is associated with the skew scattering, while the second is the side-jump contribution. Notice that the side-jump conductivity $-\lambda / 2 \rho_{D}=$ $-\alpha n e^{2}$ depends neither on the strength of disorder nor on the strength of the electron-electron interaction. In contrast, the spin-Coulomb drag reduces by factor $1+\rho^{S D} / \rho^{D}$ the skewscattering term, so the total spin-Hall conductivity and the spin-Hall current are reduced as well.

The temperature dependence of the spin conductivities $\sigma_{y x}=j_{y}^{s z} / E_{x}$ for two different mobilities and for the parameters of the recent experiment on the $2 \mathrm{DEG}$ in $\mathrm{Al}_{0.1} \mathrm{Ga}_{0.9} \mathrm{As}$ (Ref. 11) is presented in Fig. 2. We used Eqs. (A21) and
(A22) to calculate the scattering rates. The procedure is as follows. First we find the scattering rate $\tau_{\sigma}\left(=\tau_{-\sigma}\right)$ from the mobility. Then using Eqs. (A21) and (26) we estimate the strength of impurity potential $V_{0}$. Finally, we compute the skew-scattering rate by numerically evaluating Eq. (A22). In general the skew-scattering rate of Eq. (26) is temperature dependent through the Fermi distribution. However, in a wide range of mobilities the impurity potential $V_{0}$ is much smaller than the Fermi energy and the scattering rate does not change with energy around $E_{F}$, i.e., $\left[\partial W^{a}(\epsilon, \Theta) / \partial \epsilon\right]_{\epsilon=E_{F}}$ $\approx 0$ (see Fig. 1). Since the temperature dependence of the skew-scattering rate comes from the energy dependence of $W^{a}(\epsilon, \Theta)$, we see that the temperature dependence of the skew-scattering term is very weak. In Fig. $2, \sigma_{y x}^{s j}$ is the sidejump contribution to the conductivity in the absence of electron-electron interactions (found from the last term of Eq. (32)). $\sigma_{y x}^{s s}$ is the skew-scattering conductivity in the absence of electron-electron interactions [found from second term of Eq. (32)]. $\sigma_{y x}^{t o t}$ is the total off-diagonal conductivity modified by the spin-Coulomb drag term, as given by Eq. (45). For $T=0$ the total spin conductivity is the sum of two contributions: skew-scattering proportional to $\rho_{s s} / 2 \rho_{D}^{2}$ and side-jump proportional to $\lambda / 2 \rho_{D}=-\alpha n e^{2}$. For an attractive electron-impurity potential $\left(V_{0}<0\right)$ the two contributions have opposite signs, consistent with previous theoretical estimates in Refs. 13 and 16. The ratio of the skew-scattering to side-jump terms depends on the mobility, $\bar{\mu}$. The skewscattering conductivity scales as $\bar{\mu}$ while the side-jump is independent of mobility. As a consequence the skewscattering conductivity dominates for high mobilities while the side-jump dominates at low mobilities. The different ratios of skew-scattering to side-jump conductivities reported in recent theoretical papers ${ }^{13,16}$ result from choosing different mobilities (see also Fig. 2). The spin-Coulomb drag is the only temperature-dependent contribution in this calculations: it scales as $T^{2} \ln T$ for $T<T_{F}$ and reduces the absolute value of spin conductivity and spin current. Moreover, the reduction of spin-Hall effect by spin-Coulomb drag depends strongly on the Drude resistivity. Hence the reduction of spin-Hall conductivity will be of the order of a few percent for low mobilities (invisible change in $\sigma_{y x}$ for $\bar{\mu}$ $=0.1 \mathrm{~m}^{2} / \mathrm{Vs}$ in Fig. 2) and of the order of $25 \%-50 \%$ for high mobilities (see $\sigma_{y x}$ for $\bar{\mu}=1 \mathrm{~m}^{2} / \mathrm{Vs}$ in Fig. 2).

Thus far, as stated in the Introduction, we have only considered the extrinsic spin-Hall effect. What about the intrinsic effect? In recent experiments by Sih et al. ${ }^{11}$ on a $2 \mathrm{DEG}$ confined in an (110) AlGaAs quantum well, three different contributions to the spin-Hall conductivity are present in principle: the impurities, the linear-in- $\vec{k}$ SO Rashba field, and the cubic-in- $\vec{k}$ SO Dresselhaus field which is perpendicular to the Rashba field. Since in (110) quantum wells, the Dresselhaus field is in the [110] direction; we do not expect any spin-Hall current or spin-Hall accumulation connected with this term in the plane of the quantum well. Further, it has been established that the spin-Hall conductivity in an infinite 2DEG with only linear spin-orbit interactions of Rashba or Dresselhaus type vanishes for arbitrarily weak disorder. ${ }^{40-43}$ Hence we believe that the extrinsic contribution $\sigma_{y x} \sim 2$ $\times 10^{-4} 1 / k \Omega$ is the dominant one in the referenced paper. 
Also, the theoretical estimates of extrinsic ${ }^{13}$ and intrinsic ${ }^{14}$ contributions in the experiments on three-dimensional $n$-doped GaAs (Ref. 10) show that the extrinsic contribution is an order of magnitude larger than the intrinsic one.

\section{SPIN ACCUMULATIONS}

Let us now study the influence of spin-Coulomb drag on the spin accumulation. This is relevant to the interpretation of the experiments by Sih et al. ${ }^{11}$ and Kato et al. ${ }^{10}$ We consider a very long conductor in the form of a bar of length $L$ in the $x$ direction and narrow width $W$ in the $y$ direction. A charge current flows only in the $x$ direction. The $y$ components of the current $j_{\sigma}^{y}$, with $\sigma=\uparrow$ and $\downarrow$ add up to zero everywhere and individually vanish on the edges of the system, i.e., $j_{\sigma}^{y}=0$ at $y= \pm W / 2$. In order to satisfy the boundary conditions the system cannot remain homogeneous in the $y$ direction. A position-dependent spin density, known as spin accumulation develops across the bar, and is reflected in nonuniform chemical potentials $\mu_{\sigma}(y)$. In the steady-state regime the spatial derivative of the spin current in the $y$ direction must exactly balance the relaxation of the spin density due to spin-flip processes. This condition leads to the following equation for the spin chemical potentials: ${ }^{44}$

$$
\frac{d^{2}\left[\mu_{\sigma}(y)-\mu_{-\sigma}(y)\right]}{d^{2} y}=\frac{\mu_{\sigma}(y)-\mu_{-\sigma}(y)}{L_{s}^{2}},
$$

where $L_{s}$ is the spin diffusion length. The solution of this equation is

$$
\mu_{\sigma}(y)-\mu_{-\sigma}(y)=C e^{y / L_{s}}+C^{\prime} e^{-y / L_{s}},
$$

where $\mathrm{C}, C^{\prime}$ are constants to be determined by the boundary conditions $j_{ \pm \sigma}^{y}( \pm W / 2)=0$. The effective electric field in the $y$ direction is $e E_{\sigma}^{y}=d \mu_{\sigma} / d y$. Thus,

$$
-e\left(E_{\uparrow}^{y}-E_{\downarrow}^{y}\right)=\frac{d\left(\mu_{\downarrow}-\mu_{\uparrow}\right)}{d y}=\frac{C^{\prime}}{L_{s}} e^{-y / L_{s}}-\frac{C}{L_{s}} e^{y / L_{s}} .
$$

Using the boundary conditions at $y= \pm W / 2$ we obtain

$$
\begin{aligned}
& E_{\uparrow}^{y}( \pm W / 2)=\rho_{\uparrow \uparrow}^{y x} j_{\uparrow}^{x}+\rho_{\uparrow \downarrow}^{y x} j_{\downarrow}^{x}, \\
& E_{\downarrow}^{y}( \pm W / 2)=\rho_{\downarrow \uparrow}^{y x} j_{\uparrow}^{x}+\rho_{\downarrow \downarrow}^{y x} j_{\downarrow}^{x} .
\end{aligned}
$$

Making use of Eqs. (48)-(50) to eliminate the electric field, we obtain the following set of equations:

$$
\begin{aligned}
\frac{C^{\prime}}{L_{s}} e^{-W / 2 L_{s}}-\frac{C}{L_{s}} e^{W / 2 L_{s}}= & \frac{C^{\prime}}{L_{s}} e^{W / 2 L_{s}}-\frac{C}{L_{s}} e^{-W / 2 L_{s}}=-e\left[\rho_{\uparrow \uparrow}^{y x} j_{\uparrow}^{x}\right. \\
& \left.+\rho_{\uparrow J_{\downarrow}^{y x}}^{y x}-\rho_{\downarrow \uparrow}^{y x} j_{\uparrow}^{x}-\rho_{\downarrow \downarrow}^{y x} j_{\downarrow}^{x}\right],
\end{aligned}
$$

which gives immediately the solution $C=-C^{\prime}$ and

$$
C^{\prime}=\frac{-e L_{s}\left[\rho_{\uparrow}^{y x} j_{\uparrow}^{x}+\rho_{\uparrow j}^{y x} j_{\downarrow}^{x}-\rho_{\downarrow \uparrow}^{y x} j_{\uparrow}^{x}-\rho_{\downarrow \downarrow}^{y x} j_{\downarrow}^{x}\right]}{2 \cosh \left(W / 2 L_{s}\right)} .
$$

Thus, the formula for the spin accumulation is

$$
\mu_{\uparrow}(y)-\mu_{\downarrow}(y)=\frac{e L_{s}\left[\rho_{\uparrow \uparrow}^{y x} j_{\uparrow}^{x}+\rho_{\uparrow \downarrow}^{y x} j_{\downarrow}^{x}-\rho_{\downarrow \uparrow}^{y x} j_{\uparrow}^{x}-\rho_{\downarrow \downarrow}^{y x} j_{\downarrow}^{x}\right] \sinh \left(y / L_{s}\right)}{\cosh \left(W / 2 L_{s}\right)} .
$$

Finally, upon substituting the matrix elements of the resistivity from Eq. (39) we find

$$
\mu_{\uparrow}(y)-\mu_{\downarrow}(y)=\frac{-e L_{s}\left[j_{\uparrow}^{x}\left(\rho_{\uparrow}^{s s}-\lambda_{\uparrow} \rho_{\uparrow}^{D}+A_{\uparrow}^{\gamma \alpha}+B_{\uparrow}^{\gamma \alpha}\right)+j_{\downarrow}^{x}\left(\rho_{\downarrow}^{s s}-\lambda_{\downarrow} \rho_{\downarrow}^{D}+A_{\downarrow}^{\gamma \alpha}+B_{\downarrow}^{\gamma \alpha}\right)\right] \sinh \left(y / L_{s}\right)}{\cosh \left(W / 2 L_{s}\right)} .
$$

The spin-Coulomb drag modifies the spin accumulation in three different ways: (i) the spin-Coulomb drag resistivity appears directly in the terms $A^{\gamma \alpha}$ and $B^{\gamma \alpha}$, defined after Eq. (39); (ii) the values of the spin components of the longitudinal current $j_{\sigma}^{x}$ are, in general, affected by the SCD; and (iii) the spin diffusion length $L_{s}$ is significantly reduced by the spin-Coulomb drag ${ }^{35}$ as seen from the formula ${ }^{34}$

$$
L_{s}=\frac{\chi_{0}}{\chi_{s}} \frac{L_{c}}{1+\rho_{S D} / \rho_{D}}
$$

where $\chi_{0}$ is the susceptibility, $\chi_{S}$ is the spin susceptibility, and $L_{c}$ is the density diffusion length.

In paramagnetic materials Eq. (54) simplifies to

$$
\begin{aligned}
\mu_{\uparrow}(y)-\mu_{\downarrow}(y) & =\frac{-2 e L_{s} j_{x}\left[\rho^{s s}-\lambda \rho^{D}-\lambda \rho_{S D}\right] \sinh \left(y / L_{s}\right)}{\cosh \left(W / 2 L_{s}\right)} \\
& =\frac{-2 e L_{s} E_{x}\left[\rho^{s s}-\lambda \rho^{D}-\lambda \rho_{S D}\right] \sinh \left(y / L_{s}\right)}{\rho_{D} \cosh \left(W / 2 L_{s}\right)},
\end{aligned}
$$

where $j_{x}=E_{x} / \rho_{D}$ indeed is independent of $\rho_{S D}$. Further the Eq. (56) at the edges of sample for $L=W / 2$ gives

$$
\begin{aligned}
\mu_{\uparrow}(W / 2)-\mu_{\downarrow}(W / 2)= & -2 e L_{s} j_{x}\left[\rho^{s s}-\lambda \rho^{D}\right. \\
& \left.-\lambda \rho_{S D}\right] \tanh \left(W / 2 L_{s}\right) .
\end{aligned}
$$

The three terms in the square bracket of Eq. (57) are the skew-scattering term, the side-jump contribution, and the electron-electron correction. The last term reduces the spin accumulations. Additionally, the spin-Coulomb drag reduces the spin accumulation through the spin-diffusion length [see 
Eq. (55)]. However, in the limit of $W \ll L_{s}, \tanh \left(W / 2 L_{s}\right)$ can be approximated by $W / 2 L_{s}$, and the spin accumulation at the edges becomes independent of $L_{s}$. In this limit, the influence of SCD on the spin accumulation is only through the $\lambda \rho_{S D}$ term. Notice that in the limit of infinite spin-relaxation time $\left(L_{s} \rightarrow \infty\right)$ the spin accumulation can be obtained directly from the homogeneous formulas, Eqs. (43) and (44).

For a two-dimensional electron gas confined in $\mathrm{Al}_{0.1} \mathrm{Ga}_{0.9} \mathrm{As}$ quantum well measured by $\mathrm{Sih}$ et al. ${ }^{11}$ with electron and impurity concentrations $n_{i}=n_{2 D}=2 \times 10^{12} \mathrm{~cm}^{-2}$, mobility $\bar{\mu}=0.1 \mathrm{~m}^{2} / \mathrm{Vs}, \quad L_{s}=1 \mu \mathrm{m}, \quad \tau=4 \times 10^{-5} \mathrm{~ns}, \quad \tau_{s s}$ $=0.02 \mathrm{~ns}, \alpha \hbar=4.4 \AA$ and for the sample with width $W$ $=100 \mu \mathrm{m}$, we calculate the spin accumulation to be $-1.5 \mathrm{meV} /|e|$ on the left edge of the sample (relative to the direction of the electric field), i.e., for $W / 2=-50 \mu \mathrm{m}$. This means that the nonequilibrium spin-density points down on the left edge of the sample and up on the right edge. It is not clear at present whether this sign of the spin accumulation is consistent or not with the sign of the Kerr rotation angle measured in the experiments. ${ }^{11,10}$

\section{SUMMARY}

We have developed the microscopic theory of the extrinsic spin-Hall effect taking into account the skew-scattering, side-jump, and Coulomb interaction effects. The total spin conductivity in zero temperature is a sum of the skewscattering and side-jump terms. The spin-Coulomb drag is the only temperature-dependent term, causing a quadratic-in$T$ reduction of the spin-Hall conductivity. Further, we find that the spin-Hall conductivity associated with the side-jump contribution is independent of the strength of electronelectron interactions, while the part of the spin conductivity connected with the skew scattering is reduced by the spinCoulomb drag for experimentally accessible mobilities. Moreover, we predict that in paramagnetic systems the spinCoulomb drag reduces the spin accumulations in two different ways: (i) directly through the reduction of the skewscattering contribution, and (ii) indirectly through the reduction of the spin-diffusion length.

\section{ACKNOWLEDGMENTS}

This work was supported by NSF Grant No. DMR0313681. We gratefully acknowledge valuable discussions with Shufeng Zhang, Shoucheng Zhang, Masaru Onoda, and Roland Winkler. We thank B. I. Halperin and E. Rashba for their criticism of an earlier version of this paper and for pointing out to us Ref. 37.

\section{APPENDIX: AN EXACTLY SOLVABLE MODEL FOR SKEW SCATTERING}

We present the calculations of the scattering rates for a circular well potential of the form

$$
V(r)=V_{0} \theta(a-r)+\bar{\alpha} a L_{z} S_{z} \delta(r-a) V_{0},
$$

which is attractive for $V_{0}<0$ and repulsive for $V_{0}>0$. The second term on the right is the spin-orbit interaction and $\bar{\alpha}$ $=\alpha \hbar / a^{2}$ where $\alpha$ is an effective spin-orbit coupling found in the eight-band Kane model and $a$ is the impurity radius. Since the orbital angular momentum $L_{z}=l$ and the spin angular momentum $S_{z}=\sigma$ are conserved we can separate the wave function into radial and orbital parts

$$
\Psi_{k l \sigma}(r, \theta)=R_{k l \sigma}(r) e^{i l \theta},
$$

and the corresponding Schrödinger equation has the form

$$
-\frac{\hbar^{2}}{2 m}\left(R_{k l \sigma}^{\prime \prime}+\frac{1}{r} R_{k l \sigma}^{\prime}\right)+\frac{\hbar^{2} l^{2}}{2 m r^{2}} R_{k l \sigma}+V(r) R_{k l \sigma}(r)=E R_{k l \sigma}(r),
$$

where $E=\hbar^{2} k^{2} / 2 m$ and the prime denotes a derivative with respect to $r$. We now express lengths and wave vectors in units $a$ and $a^{-1}$ respectively, so $r$ should be understood as $r / a, k$ as $k a$, and, of course, $a=1$ in these units. The dimensionless Schrödinger equation is

$$
R_{k l \sigma}^{\prime \prime}+\frac{1}{r} R_{k l \sigma}^{\prime}+\left(k^{2}-v_{0}-\frac{l^{2}}{r^{2}}\right) R_{k l \sigma}=0, \quad r<1,
$$

and

$$
R_{k l \sigma}^{\prime \prime}+\frac{1}{r} R_{k l \sigma}^{\prime}+\left(k^{2}-\frac{l^{2}}{r^{2}}\right) R_{k l \sigma}=0, \quad r>1,
$$

where $v_{0}=2 m V_{0} a^{2} / \hbar^{2}$ is the dimensionless parameter which measures the height of the impurity potential barrier. The regular solution of this equation for $r<1$ is

$$
R_{k l \sigma}(r)=J_{|l|}(\nu r), \quad r<1,
$$

where we have defined $\nu=\sqrt{k^{2}-v_{0}}$. On the other hand, the solution for $r>1$ can be written as a superposition of the two independent solutions of the differential equation (A5),

$$
R_{k l \sigma}(r)=e^{i \delta_{l \sigma}\left[\cos \delta_{l \sigma} J_{|l|}(k r)-\sin \delta_{l \sigma} Y_{|l|}(k r)\right], \quad r>1 .}
$$

The matching conditions on the wave function and its derivative lead to the following equations:

$$
\begin{gathered}
R_{k l \sigma}\left(1^{+}\right)=R_{k l \sigma}\left(1^{-}\right)=R_{k l \sigma}(1), \\
R_{k l \sigma}^{\prime}\left(1^{+}\right)-R_{k l \sigma}^{\prime}\left(1^{-}\right)=\bar{\alpha} l \sigma R_{k l \sigma}(1) v_{0} .
\end{gathered}
$$

Substitution of Eqs. (A6) and (A7) to the matching conditions yields

$$
\frac{k \cos \delta_{l \sigma} J_{|l|}^{\prime}(k)-k \sin \delta_{l \sigma} Y_{|l|}^{\prime}(k)}{\cos \delta_{l \sigma} J_{|l|}(k)-\sin \delta_{l \sigma} Y_{|l|}(k)}=\nu \frac{J_{|l|}^{\prime}(\nu)}{J_{|l|}(\nu)}+\bar{\alpha} l \sigma v_{0},
$$

from which one gets the following equation for the phase shifts $\delta_{l \sigma}$ :

$$
\cot \delta_{l \sigma}=\frac{k Y_{|l|}^{\prime}(k)-\beta_{l \sigma} Y_{|l|}(k)}{k J_{|l|}^{\prime}(k)-\beta_{l \sigma} J_{|l|}(k)},
$$

where $\beta_{l \sigma}=\nu J_{|l|}^{\prime}(\nu) / J_{|l|}(\nu)+\bar{\alpha} l \sigma v_{0}, J_{|l|}(k)$, and $Y_{|l|}(k)$ are the Bessel functions of the first and second kinds. The wave 
function at large distance from the scattering center can be written as

$$
\begin{aligned}
\psi_{k l \sigma}(r, \theta) & \sim \sqrt[r \rightarrow \infty]{\frac{2}{\pi k r}} \cos \left(k r-\frac{|l| \pi}{2}-\frac{\pi}{4}+\delta_{l \sigma}\right) e^{i l \theta} e^{i \delta_{l \sigma}} \\
& =\psi_{k l \sigma}^{0}(r, \theta)+\frac{e^{2 i \delta_{l \sigma}-1}}{\sqrt{2 \pi k r}} e^{i(k r-|l| \pi / 2-\pi / 4)} e^{i l \theta}, \quad \mathrm{A}
\end{aligned}
$$

where $\psi_{k l \sigma}^{0}(r, \theta)=\sqrt{\frac{2}{\pi k r}} \cos \left(k r-\frac{|l| \pi}{2}-\frac{\pi}{4}\right) e^{i l \theta}$ is the free wave function in the channel of angular momentum $l$. The scattering amplitude $f_{\sigma}(k, \theta)$ is the factor multiplying the outgoing wave $e^{i k r} / \sqrt{r}$ in the above equation,

$$
f_{\sigma}(k, \theta)=\sum_{l=-\infty}^{\infty} \frac{e^{2 i \delta_{l \sigma}-1}}{\sqrt{2 \pi k}} e^{-i(|l| \pi / 2+\pi / 4)} e^{i l \theta} .
$$

The differential cross section is accordingly given by

$$
\begin{aligned}
\left(\frac{d \sigma_{c}}{d \theta}\right)_{\sigma}= & \left|f_{\sigma}(k, \theta)\right|^{2}=\frac{1}{2 \pi k} \sum_{l, l^{\prime}}\left(e^{\left.2 i \delta_{l \sigma}-1\right)}\right. \\
& \times\left(e^{\left.-2 i \delta_{l^{\prime} \sigma}-1\right)} e^{-i \pi / 2\left(|l|-\left|l^{\prime}\right|\right)} e^{i\left(l-l^{\prime}\right) \theta} .\right.
\end{aligned}
$$

Finally we notice that the total scattering rate is related to the differential scattering cross section for a single impurity as follows:

$$
W(k, \theta)=W^{s}(k, \theta)+\sigma W^{a}(k, \theta) \sin \theta=n_{i} \frac{4 \pi^{2} \hbar^{3} k}{m^{2} \mathcal{A}} \frac{d \sigma_{c}}{d \theta},
$$

where $n_{i}=N_{i} / \mathcal{A}$ is the areal density of impurities. Combining this with Eq. (A14) we find

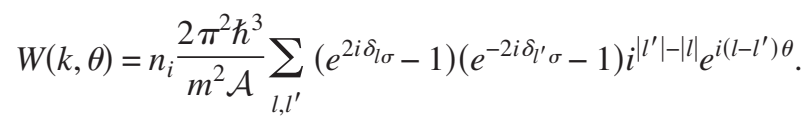

To identify $W^{s}$ and $W^{a}$ we separate Eq. (A16) into even and odd components with respect to the scattering angle $\theta$, which can be easily done using the identity $e^{i\left(l-l^{\prime}\right) \theta}=\cos \left[\left(l-l^{\prime}\right) \theta\right]$ $+i \sin \left[\left(l-l^{\prime}\right) \theta\right]$. Then

$$
\begin{aligned}
W^{s}(k, \theta)= & n_{i} \frac{2 \pi^{2} \hbar^{3}}{m^{2} \mathcal{A}} \sum_{l, l^{\prime}}\left(e ^ { 2 i \delta _ { l \sigma } - 1 ) } \left(e^{\left.-2 i \delta_{l^{\prime} \sigma}-1\right) i i^{\left|l^{\prime}\right|-|l|}}\right.\right. \\
& \times \cos \left[\left(l-l^{\prime}\right) \theta\right]
\end{aligned}
$$

and

$$
\begin{aligned}
W^{a}(k, \theta)= & \sigma n_{i} \frac{2 \pi^{2} \hbar^{3}}{m^{2} \mathcal{A} \sin \theta} \sum_{l, l^{\prime}}\left(e^{\left.2 i \delta_{l \sigma}-1\right)}\right. \\
& \times\left(e^{\left.-2 i \delta_{l^{\prime} \sigma}-1\right) i i^{\left|l^{\prime}\right|-|l|+1} \sin \left[\left(l-l^{\prime}\right) \theta\right] .}\right.
\end{aligned}
$$

Making use of the identities $e^{ \pm 2 i \delta_{l \sigma}-1}= \pm 2 i /\left(\cot \delta_{l \sigma} \mp i\right)$ we rewrite the scattering rates as

$$
W^{s}(k, \theta)=n_{i} \frac{8 \pi^{2} \hbar^{3}}{m^{2} \mathcal{A}} \sum_{l, l^{\prime}} \frac{i^{\left|l^{\prime}\right|-|l|} \cos \left[\left(l-l^{\prime}\right) \theta\right]}{\left(\cot \delta_{l \sigma}-i\right)\left(\cot \delta_{l^{\prime} \sigma}+i\right)}
$$

and

$$
W^{a}(k, \theta)=\sigma n_{i} \frac{8 \pi^{2} \hbar^{3}}{m^{2} \mathcal{A}} \sum_{l, l^{\prime}} \frac{i^{\left|l^{\prime}\right|-|l|+1} \sin \left[\left(l-l^{\prime}\right) \theta\right]}{\left(\cot \delta_{l \sigma}-i\right)\left(\cot \delta_{l^{\prime} \sigma}+i\right) \sin \theta},
$$

where the phase shifts are completely determined by Eq. (A11). Notice that the sums over $l$ and $l^{\prime}$ in Eq. (A19) and Eq. (A20) run from $-\infty$ to $\infty$ and the phase shifts have the symmetries $\delta_{-l,-\sigma}(\alpha)=\delta_{l, \sigma}(\alpha)$ and $\delta_{-l, \sigma}(-\alpha)=\delta_{l, \sigma}(\alpha)$, which implies that $W^{s}(k, \theta)$ and $W^{a}(k, \theta)$ are invariant under spin reversal $\sigma \rightarrow-\sigma$ and $W^{a}(k, \theta)$ changes sign with a change of sign of $\alpha$, as expected. The integral over $\theta$ Eq. (28) eliminates the majority of the terms from the sum over $l$ and $l^{\prime}$ and the only nonzero terms for $W^{s}(k, \theta)$ are with $l=l^{\prime}$ and $l$ $=l^{\prime} \pm 1$. This gives

$$
\begin{aligned}
\int_{0}^{2 \pi} d \theta W^{s}(k, \theta)(1-\cos \theta)= & n_{i} \frac{8 \pi^{3} \hbar^{3}}{m^{2} \mathcal{A}}\left\{\sum_{l} \frac{2}{\left(\cot \delta_{l \sigma}^{2}+1\right)}\right. \\
& \left.-\sum_{l=l^{\prime} \pm 1} \frac{i^{\left|l^{\prime}\right|-|l|}}{\left(\cot \delta_{l \sigma}-i\right)\left(\cot \delta_{l^{\prime} \sigma}+i\right)}\right\} .
\end{aligned}
$$

One can see that $W^{s}(k, \theta)$ is modified by spin-orbit interactions, however it has a nonzero value even if spin-orbit interactions are absent. We checked numerically that the sum over $l$ in Eq. (A21) is convergent after taking into account a few first terms. For the skew-scattering rate $W^{a}(k, \theta)$, the integral over $\theta$ Eq. (29) have nonzero terms only if $l=l^{\prime} \pm 1$. This gives

$$
\begin{aligned}
\int_{0}^{2 \pi} d \theta W^{a}(k, \theta) \sin ^{2} \theta= & \sigma n_{i} \frac{8 \pi^{3} \hbar^{3}}{m^{2} \mathcal{A}} \\
& \times \sum_{l=l^{\prime} \pm 1} \frac{i i^{l^{\prime}|-| l \mid+1}}{\left(\cot \delta_{l \sigma}-i\right)\left(\cot \delta_{l^{\prime} \sigma}+i\right)} .
\end{aligned}
$$

For very small $k a$ the only relevant terms are $l^{\prime}=0,|l|=1$ and $\left|l^{\prime}\right|=1, l=0$ which yields to

$$
\int_{0}^{2 \pi} d \theta W^{a}(k, \theta) \sin ^{2} \theta \simeq \sigma n_{i} \frac{8 \pi^{3} \hbar^{3}}{m^{2} \mathcal{A}}\left[\frac{1}{\left(\cot \delta_{1 \sigma}-i\right)\left(\cot \delta_{0 \sigma}+i\right)}\right.
$$

$$
\begin{aligned}
& -\frac{1}{\left(\cot \delta_{0 \sigma}-i\right)\left(\cot \delta_{-1 \sigma}+i\right)} \\
& +\frac{1}{\left(\cot \delta_{0 \sigma}-i\right)\left(\cot \delta_{1 \sigma}+i\right)} \\
& -\frac{1}{\left(\cot \delta_{-1 \sigma}-i\right)\left(\cot \delta_{0 \sigma}+i\right)}
\end{aligned}
$$




$$
\begin{aligned}
= & \sigma n_{i} \frac{16 \pi^{3} \hbar^{3}}{m^{2} \mathcal{A}} \\
& \times\left[\operatorname{Re} \frac{1}{\left(\cot \delta_{1 \sigma}-i\right)\left(\cot \delta_{0 \sigma}+i\right)}\right. \\
& \left.-\operatorname{Re} \frac{1}{\left(\cot \delta_{-1 \sigma}-i\right)\left(\cot \delta_{0 \sigma}+i\right)}\right] \\
= & \sigma n_{i} \frac{16 \pi^{3} \hbar^{3}}{m^{2} \mathcal{A}}
\end{aligned}
$$

$$
\begin{aligned}
& \times\left[\frac{1+\cot \delta_{0 \sigma} \cot \delta_{1 \sigma}}{\left(\cot ^{2} \delta_{1 a}+1\right)\left(\cot ^{2} \delta_{0 \sigma}+1\right)}\right. \\
& \left.-\frac{1+\cot \delta_{0 \sigma} \cot \delta_{-1 \sigma}}{\left(\cot ^{2} \delta_{-1 \sigma}+1\right)\left(\cot ^{2} \delta_{0 \sigma}+1\right)}\right],
\end{aligned}
$$

independent of $\theta$. Notice that $\int_{0}^{2 \pi} d \theta W^{a}(k, \theta) \sin ^{2} \theta$ vanishes in the absence of spin-orbit interactions, since all the phase shifts are independent of $\sigma$ in that case.
*Electronic address: vignaleg@missouri.edu

${ }^{1}$ S. A. Wolf, D. D. Awschalom, R. A. Buhrman, J. M. Duaghton, S. von Molnar, M. L. Rouke, A. Y. Chtchelkanova, and D. M. Treger, Science 294, 1488 (2001).

${ }^{2}$ M. I. Dyakonov and V. I. Perel, Phys. Lett. 35, 459 (1971).

${ }^{3}$ M. I. Dyakonov and V. I. Perel, Zh. Eksp. Teor. Fiz. 13, 657 (1971).

${ }^{4}$ J. E. Hirsch, Phys. Rev. Lett. 83, 1834 (1999).

${ }^{5}$ S. Zhang, Phys. Rev. Lett. 85, 393 (2000).

${ }^{6}$ S. Murakami, N. Nagaosa, and S.-C. Zhang, Science 301, 1348 (2003)

${ }^{7}$ J. Sinova, D. Culcer, Q. Niu, N. A. Sinitsyn, T. Jungwirth, and A. H. MacDonald, Phys. Rev. Lett. 92, 126603 (2004).

${ }^{8}$ S. Murakami, Adv. Solid State Phys. 45, 197 (2005).

${ }^{9}$ E. I. Rashba, Phys. Rev. B 68, 241315(R) (2003).

${ }^{10}$ Y. K. Kato, R. C. Myers, A. C. Gossard, and D. D. Awschalom, Science 306, 1910 (2004).

${ }^{11}$ V. Sih, R. C. Myers, Y. K. Kato, W. H. Lau, A. C. Gossard, and D. D. Awschalom, Nat. Phys. 1, 31 (2005).

${ }^{12}$ J. Wunderlich, B. Kaestner, J. Sinova, and T. Jungwirth, Phys. Rev. Lett. 94, 047204 (2005).

${ }^{13}$ H.-A. Engel, B. I. Halperin, and E. Rashba, Phys. Rev. Lett. 95, 166605 (2005).

${ }^{14}$ B. A. Bernevig and S.-C. Zhang, cond-mat/0412550.

${ }^{15}$ B. K. Nikolic, S. Souma, L. P. Zarbo, and J. Sinova, Phys. Rev. Lett. 95, 046601 (2005).

${ }^{16}$ W.-K. Tso and S. D. Sarma, Phys. Rev. Lett. 96, 056601 (2006).

${ }^{17}$ W.-K. Tse, J. Fabian, I. Zutic, and S. D. Sarma, Phys. Rev. B 72, 241303 (2005).

${ }^{18}$ P. Noziéres and C. Lewiner, J. Phys. (Paris) 34, 901 (1973).

${ }^{19}$ J. Smit, Physica (Amsterdam) 21, 877 (1955).

${ }^{20}$ J. Smit, Physica (Amsterdam) 24, 39 (1958).

${ }^{21}$ N. F. Mott and H. S. W. Massey, The Theory of Atomic Collisions (Oxford University Press, 1964).

${ }^{22}$ L. Berger, Phys. Rev. B 2, 4559 (1970).
${ }^{23}$ L. Berger, Phys. Rev. B 5, 1862 (1972).

${ }^{24}$ S. K. Lyo and T. Holstein, Phys. Rev. Lett. 29, 423 (1972).

${ }^{25}$ R. Karplus and J. M. Luttinger, Phys. Rev. 95, 1154 (1954).

${ }^{26}$ V. K. Dugaev, A. Crépieux, and P. Bruno, Phys. Rev. B 64, 104411 (2001).

${ }^{27}$ A. Crépieux and P. Bruno, Phys. Rev. B 64, 014416 (2001).

${ }^{28}$ T. Jungwirth, Q. Niu, and A. H. MacDonald, Phys. Rev. Lett. 88, 207208 (2002).

${ }^{29}$ M. Onoda and N. Nagaosa, Phys. Rev. Lett. 90, 206601 (2003).

${ }^{30}$ V. K. Dugaev, P. Bruno, M. Taillefumier, B. Canals, and C. Lacroix, Phys. Rev. B 71, 224423 (2005).

${ }^{31}$ N. A. Sinitsyn, Q. Niu, J. Sinova, and K. Nomura, Phys. Rev. B 72, 045346 (2005).

${ }^{32}$ I. D'Amico and G. Vignale, Phys. Rev. B 62, 4853 (2000).

${ }^{33}$ K. Flensberg, T. S. Jensen, and N. A. Mortensen, Phys. Rev. B 64, 245308 (2001).

${ }^{34}$ I. D'Amico and G. Vignale, Phys. Rev. B 65, 085109 (2002).

${ }^{35}$ C. P. Weber, N. Gedik, J. E. Moore, J. Orenstein, J. Stephens, and D. D. Awschalom, Bull. Am. Phys. Soc. 50, 1320 (2005).

${ }^{36} \mathrm{R}$. Winkler, Spin-Orbit Effects in Two-Dimensional Electron and Hole Systems (Springer, New York, 2003).

${ }^{37}$ W. Kohn and J. M. Luttinger, Phys. Rev. 108, 590 (1957).

${ }^{38}$ L. D. Landau and E. M. Lifshitz, Course of Theoretical Physics, Vol. III (Butterworth-Heinemann, Oxford, 1964).

${ }^{39}$ I. D'Amico and G. Vignale, Phys. Rev. B 68, 045307 (2003).

${ }^{40}$ E. G. Mishchenko, A. V. Shytov, and B. I. Halperin, Phys. Rev. Lett. 93, 226602 (2004).

${ }^{41}$ A. Khaetskii, Phys. Rev. Lett. 96, 056602 (2006).

${ }^{42}$ R. Raimondi and P. Schwab, Phys. Rev. B 71, 033311 (2005).

${ }^{43}$ E. M. Hankiewicz, G. Vignale, and M. Flatté, cond-mat/0603144 (unpublished).

${ }^{44}$ T. Valet and A. Fert, Phys. Rev. B 48, 7099 (1993).

${ }^{45}$ Strictly speaking spin-flip scattering also renormalizes the ordinary momentum relaxation time $\tau_{\sigma}$. This small correction is neglected here: it can easily be taken into account if needed. 